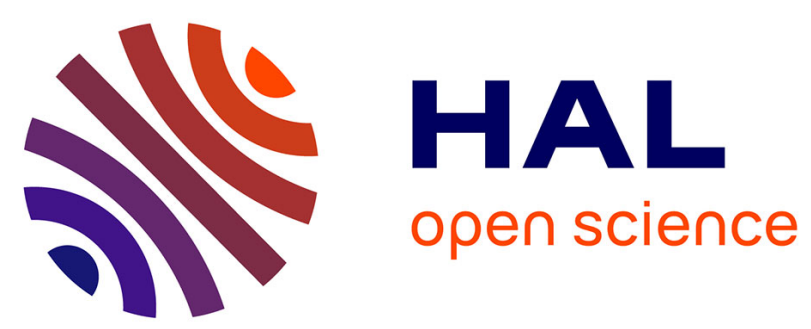

\title{
FONDATIONS MONASTIQUES ET TERRITORIALITÉ.COMMENT RODRIGUE DE TOLÈDE A INVENTÉ LA CASTILLE
}

Georges Martin

\section{- To cite this version:}

Georges Martin. FONDATIONS MONASTIQUES ET TERRITORIALITÉ.COMMENT RODRIGUE DE TOLÈDE A INVENTÉ LA CASTILLE. Patrick HENRIET. A la recherche de légitimités chrétiennes. Représentations de l'espace et du temps dans l'Espagne des IXe-XIIIe siècles., 15, ENSEditions et Casa de Velázquez, pp.243-261, 2003, Annexes des Cahiers de linguistique et de civilisation hispaniques médiévales. halshs-00114956

\section{HAL Id: halshs-00114956 \\ https://shs.hal.science/halshs-00114956}

Submitted on 19 Nov 2006

HAL is a multi-disciplinary open access archive for the deposit and dissemination of scientific research documents, whether they are published or not. The documents may come from teaching and research institutions in France or abroad, or from public or private research centers.
L'archive ouverte pluridisciplinaire HAL, est destinée au dépôt et à la diffusion de documents scientifiques de niveau recherche, publiés ou non, émanant des établissements d'enseignement et de recherche français ou étrangers, des laboratoires publics ou privés. 


\title{
FONDATIONS MONASTIQUES ET TERRITORIALITÉ. COMMENT RODRIGUE DE TOLÈDE A INVENTÉ LA CASTILLE
}

\author{
Georges MARTIN \\ École Normale Supérieure \\ Lettres et Sciences Humaines \\ GDR 2378 - SIREM (CNRS)
}

Dans le cinquième livre de son Historia de rebus Hispaniae ${ }^{1}$, oeuvre achevée pour l'essentiel en 1243 et dédiée au roi de Castille et de León Ferdinand III, Rodrigue Jimenez de Rada, archevêque de Tolède et primat de l'Église d'Espagne, évoque trois œuvres pieuses qu'il attribue à des seigneurs castillans : la fondation de Saint-Pierre d'Arlanza par le comte Ferrand Gonzalez, celle de Saint-Côme et Saint-Damien de Covarrubias par le comte Garsias Fernandez, fils et successeur de Ferrand Gonzalez, celle enfin du monastère d'Oña par le comte Sanche Garsias, fils et successeur de Garsias Fernandez ${ }^{2}$.

Ces fondations, leurs auteurs, les dotations qu'elles reçurent et, à l'occasion, les circonstances qui les environnèrent, il est peu probable que Rodrigue en ait eu vent par l'historiographie. La principale source du Tolédan, le Chronicon mundi ${ }^{3}$, achevé en 1236, et dont l'auteur, le Léonais Luc de Túy, se montre pourtant très attentif aux fondations monastiques des rois de León, n'en garde pas la mémoire. Dans le Chronicon, la curiosité du Tolédan quant aux premiers comtes castillans ne pouvait guère se satisfaire que de conquêtes et de fondations profanes, cette fois profusément répertoriées: peuplement d'Amaya par le comte Rodrigue, de Burgos par le comte Diègue, de Rueda par le comte Muño Muñiz, d'Osma par Gonzague Tellez, d'Aza, de Clunia et de San Esteban par Gonzague Fernandez et, pour les hommes évoqués plus

\footnotetext{
${ }^{1}$ RODERICUS XIMENIUS DE RADA, Opera, (María de los Desamparados CABANES PECOURT, éd.), Saragosse : Anubar (Textos medievales, 22), 1985 [fac-similé de l'édition de LORENZANA, Sanctorum patrum toletanorum, 3, 1793] ; Historia de rebus Hispaniae, p. 1-208; désormais De rebus. Cinquième livre : p. 97-117. Sur l'auteur et son oeuvre, voir Georges MARTIN, Les Juges de Castille. Mentalités et discours politique dans l'Espagne médiévale, Paris : Klincksieck, 1992, p. 251-316.

${ }^{2}$ De rebus, p. 99a pour les deux premières, p. 99b-100a pour la troisième.

${ }^{3}$ LUC DE TÚY, Chronicon mundi, in: Hispaniae illustratae..., (Andreas SCHOTT, éd.), 4 t. en 3 vol., Francfort, 1603-1608, 4, p. 1-119; désormais Chronicon. Sur l'auteur et son oeuvre, voir G. MARTIN, Les Juges de Castille..., p. 201-249.
} 
haut, peuplement de Sepúlveda par Ferrand Gonzalez et récupération de Gormaz, d'Osma et de San Esteban par Sanche Garsias ${ }^{4}$.

Ces dernières informations, qu'avait ignorées jusque-là l'historiographie léonaise, ou qu'elle n'avait pas jugé bon de retenir, Luc les avait sans doute trouvées soit, et c'est le plus probable, dans une version interpolée vers le milieu du XIIe siècle de la Chronique perdue de Sampire ${ }^{5}$ soit, quoique moins vraisemblablement, dans la source de cette interpolation : des Annales castellani dont la première version avait été composée vers le milieu du Xe siècle ${ }^{6}$. Mais Rodrigue aurait-il consulté à son tour les Annales castellani ou les premières Annales tolédanes ${ }^{7}$ qui traduisent en castillan leur seconde version, se serait-il même penché sur des états anciens des Éphémérides de la Rioja -les Annales compostellani ${ }^{8}$, le Chronicon burgense ${ }^{9}$, aurait-il enfin, lui dont le lignage paternel était navarrais et qui avait fait traduire à Tolède, vers 1220 , le Liber regum $^{10} \mathrm{du}$ navarrais en castillan, examiné les Chroniques navarraises ${ }^{11}$ brèves dont la production avait environné, dans le dernier quart du XIIe siècle, la composition de cette histoire généalogique des royautés d'Espagne, Rodrigue aurait-il manié tout le corpus aujourd'hui conservé de la tradition historiographique asturienne, léonaise, navarraise et castillane, sa connaissance des fondations réalisées par les comtes de Castille ne serait

\footnotetext{
${ }^{4}$ Chronicon, p. 90 pour les fondations de Sanche, p. 84 pour toutes les autres.

${ }^{5}$ Interpolation publiée par Manuel GÓMEZ-MORENO dans Discursos leídos ante la Real Academia de la Historia el día 27 de mayo de 1917, Madrid, 1917, p. 29. La Chronique de Sampire, composée au tournant des Xe et XIe siècles, nous est connue par sa compilation dans la Chronique de Pélage, l'Historia silensis, la Chronica najerensis, le Chronicon mundi, etc.

${ }^{6}$ Annales castellani I et Annales castellani II, in: M. GÓMEZ-MORENO, Discursos leídos..., p. 23-28. L'événement le plus tardif recueilli dans les premières annales est daté de 977 , l'événement le plus tardif des secondes, qui continuent les premières, est daté de 1175. Les premières annales castillanes sont connues à travers un manuscrit daté de 1058 et trouvé à Saint-Isidore de León, où Luc écrivit le Chronicon mundi (M. GÓMEZ-MORENO, p. 7). Dans le Chronicon, toutefois, l'énoncé de ces fondations est, dans sa forme, très proche de l'interpolation faite au texte de Sampire.

${ }^{7}$ Enrique FLÓREZ, España sagrada, 29 vol., Madrid, 1745-1775, 23, p. 382-401 pour les Annales tolédanes I. Seule cette première version, datant d'environ 1219, s'intéresse aux faits des premiers comtes castillans.

${ }^{8}$ Ibid., 23, p. 318-325. L'édition du Père Florez, peu fiable, il est vrai, pourrait donner à penser que les Annales compostellani connurent au moins trois grands mouvements rédactionnels : le premier dans la seconde décennie du XIIe siècle (dernière date: 1118 ; date suivante : 1077), le second au début du XIIIe (dernière date: 1208; date suivante: 1109), le troisième dans les années centrales du XIIIe (dernière date : 1248).

${ }^{9}$ Ibid., p. 308-311. Le Chronicon burgense, qui se ferme sur la bataille de Las Navas de Tolosa, pourrait avoir été écrit, en 1212, pour saluer cette importante victoire chrétienne. Certains pensent néanmoins qu'il ne constitue qu'une abréviation des Annales compostellani.

${ }^{10}$ Édition : Louis COOPER, El Liber regum. Estudio linguístico, Saragosse : Institución "Fernando el Católico", 1960. Version tolédane (édition partielle) : Enrique FLÓREZ, Memorias de las reynas católicas, 2 vol., Madrid : Antonio Marín, 1761, 1, p. 481-494. Datation révisée : MARTIN, Les Juges de Castille..., p. 46-82

${ }^{11}$ Antonio UBIETO ARTETA (éd .), Corónicas navarras, Valence : Anubar (Textos medievales, 14), 1964.
} 
guère allée au-delà des conquêtes et des peuplements dont Luc de Túy s'était fait l'écho. Tout au plus aurait-il pu apprendre, à la lecture de la rédaction la plus ancienne des Annales compostellani ou du Chronicon burgense, que le corps de Garsias Fernandez avait été ramené de Cordoue, où le comte était mort, à [Saint-Pierre de] Cardeña pour y être enterré $^{12}$, ou bien encore, cette fois par les premières Annales tolédanes, découvrir que Garsias Fernandez et son fils Sanche Garsias auraient fondé le monastère asturien de [Saint-Jean] de Corias $^{13}$, découverte suspecte, en vérité, dont notre historien, bien inspiré, n'aurait alors pas fait usage... Seule la Chronica najerensis ${ }^{14}$, composée vers 1160 dans la Rioja castillane, et dont l'auteur connaissait aussi le transport à Cardeña de la dépouille de Garsias Fernandez ${ }^{15}$, aurait pu apprendre à Rodrigue que le comte Sanche Garsias avait été " sepultus apud Onie monasterium quod fecerat" "16. Mais il n'est pas sûr que la Chronica najerensis ait compté au nombre des sources du Tolédan ${ }^{17}$. De toute façon, la moisson ne serait pas bien riche.

Rodrigue a-t-il alors été renseigné par la documentation? Dans le cartulaire d'Arlanza figure une charte que l'on tient aujourd'hui pour apocryphe, datée de 912 mais vraisemblablement forgée au XIe siècle, par laquelle Ferrand Gonzalez restaure et augmente les biens du monastère en lui donnant l'église de Saint-Pierre et Saint-Paul et $\mathrm{d}^{\prime}$ autres possessions nombreuses ${ }^{18}$. Les archives de la collégiale de Covarrubias, celles de la cathédrale de Burgos et, pour un de ces documents, celles de la cathédrale de Tolède, conservent des copies anciennes, parfois même l'original, des principales chartes de fondation du monastère de Saint-Côme et Saint-Damien. Les archives

\footnotetext{
${ }^{12}$ FLÓREZ, respectivement : p. 320 et 309.

13 "Rebelo Sancho Garcia con la tierra a su padre el Conde Garci Fernandez Era MXXXII. Estos ficieron el Monesterio que dice Gaurias Era MXXXII ", ibid., p. 384. Mauvaise lecture, sans doute, des Annales castellani II qui donnent: " [...] Obiit Piniolus comes era MLXXXVII, XI kalendas iunii. Obiit comitissa domna Ildontia VII kalendas octobris. Isti hedificaverunt monasterium quod dicitur Kaurias in era MXXXII ", GÓMEZ-MORENO, p. 28.

${ }^{14}$ Antonio UBIETO ARTETA (éd.), Crónica najerense. Estudio preliminar, edición crítica e índices, Valence : Anubar (Textos medievales, 15), 1966.

${ }^{15}$ Ibid., p. 90 [4].

${ }^{16}$ Ibid., p. 91.

${ }^{17}$ René COTRAIT, Histoire et poésie. Le comte Fernán González, Grenoble : Imprimerie Allier, 1977, p. 337 et 339. Les textes sont proches, néanmoins: "in monasterio Oniensi quod ipse construxerat est sepultus ", De rebus, p. 100 a.

${ }^{18}$ Ce document et ceux qui vont suivre ont été maintes fois publiés. Je retiens la dernière et excellente édition de la documentation des comtes de Castille qu'agrémentent d'utiles commentaires: Manuel ZABALZA DUQUE, Colección diplomática de los Condes de Castilla, Salamanque : Junta de Castilla y León, 1998. Arlanza : doc. 1, p. 113-130. Covarrubias : doc. 48, p. 380-382 et doc. 52, p. 396-407. Oña : doc. 64, p. 458-478. L'original du cartulaire d'Arlanza a disparu au cours de la guerre civile de 19361939. Le Père Luciano SERRANO l'a entièrement transcrit dans Cartulario de San Pedro de Arlanza.
} 
diocésaines de Burgos, les Archives historiques nationales renferment plusieurs copies de la charte de fondation du monastère d'Oña par le comte Sanche contemporaines des faits ou en tout cas antérieures à la composition du De rebus. Ce que rapporte Rodrigue et que l'historiographie qui le précède ignore formait donc un fonds documentaire dont notre historien-primat, directement ou indirectement, a pu avoir connaissance. Il y a là innovation, très vraisemblablement travail de recherche, et nous devons considérer que ces apports, qui ajoutent la fondation monastique à l'activité militaire et de peuplement, sont chargés d'une signification importante.

Or que nous disent les chartes du sens des fondations monastiques des comtes de Castille? Toutes, en des termes variables quoique ressemblants, expriment la même intention. Elles constituent des oeuvres pro anima, destinées à aider au salut des fondateurs ainsi, parfois, que de leurs ancêtres et de leurs descendants ${ }^{19}$. Cette vocation se traduit du reste, dans le cas de Covarrubias et d'Oña, par la “donation " à Dieu d'une fille du comte, laquelle devient le seigneur de l'établissement fondé par son père et de ses possessions, habituellement considérables ${ }^{20}$. Même s'il n'est pas interdit à l'historien

\footnotetext{
Antiguo monasterio benedictino, Madrid, 1925 (doc. $\mathrm{n}^{\circ}$ 1). Pour les autres éditions de l'apocryphe de 912, voir ZABALZA DUQUE, p. 113.

${ }^{19}$ Arlanza (a. 912, apocryphe du XIe siècle) : “ Licet primordia bonorum operum, que inspirante Deo in mente gignitur, iustitie operibus deputetur, tamen ea que maiori cumulo et pociori crescunt, ampliori remuneratione expectatur in premio. Digne, igitur, iam sue spei vota in domo celica mansionum multarum collocat, qui domum sante ecclesie restaurat vel in melius construere provocat. Nos, igitur, piaculorum nostrorum honeri spei gravationem cupientes expiari flagitia ; et peccatorum nostrorum molem orationum vestrorum desiderantes adiutorio sublevare, parva pro magnis offerimus munuscula, nulliusque idem, in hoc seculo, hominum vos indigere censemus, quia iam per sanctificationem Dei Dominus noster in suo vos regno propio ditatos munere cumulavit", ibid., p. 114. Covarrubias (a. 978) : "Hoc est series testamenti que patrari uoluimus, ego Garsea Fredinandez, cum coniuge mea Aua comitissa, extremitate ultimi iudicii diem eixius decernentes, expedit ducatum mentibus nostris aliquid preponere, quatenus cum tuba terribilis mundum concusserit omne iubamine a culpe in Christo mereamur accipere ", ibid., p. 397-398. Oña (a. 1011) : "Ego, igitur, Sancius comes, cum coniuge mea comitissa nomine Urraka, ad hanc salubrem redemptoris nostri peruenimus uocem, et cum intentis cordibus, et libero arbitrio meditando, de omnibus nostris facultatibus abstulimus et ob remedium nostrarum animarum hanc domum sancte conuersationis, ad honorem santi Saluatoris uel Marie uirginis, seu sancti Micaelis archangelis, construimus ceterumque innumerabilium sanctorum, corum reliquie hic sunt recondite, ut il ultimo iudicii die cum fulserit terror tremendi iudicii, magna in gloria contuentes eius desteram, per horum preces ac suffragia illesi, euadamus auerni suplicia. [...] Ergo, ego Santius vna cum coniuge mea Vrraca, legere audientes, adque diuine lecioni fideliter credentes, quod, sicut ignis aqua ita pecatum deletun (sic), helemosina. Villas has pernotatas nostreque dominacioni subiectas helemosinaria donacione, ad predicti loci Onie oratorium, in honore Saluatoris, Domini Nostri Ihesu Christi, consecratum, sine calumpnia, sine iniuria, sine contradicione, offerentes ad famulorum famularumque Dei seruientium, regulariter ibi degencium legali a stipulacione, in eternam posessionem donauimus, tradimus atque sancimus, tum, pro nostrum anime et corporis salute tum esciam (sic), tam per horum qui iam defunti ab hoc seculo migrauerunt parentum quam certe pro illorum, qui de nostra progenie nacituri (sic) totius carnis viam sunt ingressuri, remisione peccatorum, ea dum tasat in ita cum eis sentenciali conuencione ", ibid., p. 460 et 462.

${ }^{20}$ Pour Covarrubias, voir note 52.
} 
de percevoir, sous le couvert de motivations spirituelles, le soin, plus politique, que prit un lignage d'étendre son influence à travers des seigneuries ecclésiastiques, l'explicite des chartes renferme fondations, donations et dotations dans la clôture du rapport qui lie un homme, sa femme et son lignage à la divinité.

Bien entendu, cette vocation privée et spirituelle n'est pas ce qui intéresse annalistes et chroniqueurs. Dans leurs oeuvres, la mention d'une fondation ou d'un ensevelissement vient, selon les cas, contribuer au prestige d'un établissement religieux -mentions de Corias dans les seconds Annales castellani et les premières Annales tolédanes- ou bien, dans une perspective plus laïque, illustrer le volet ecclésial d'un gouvernement, conformant du même coup, par une sorte de cartographie des lieux de mémoire, le passé d'une communauté politique. En ce qui concerne la Castille, néanmoins, ces indications, extrêmement rares et succintes, ne constituent jusqu'à Rodrigue que des annotations incidentes et inorganiques, portées comme en marge du propos. Au sein de la tradition historiographique de l'Espagne occidentale, le De rebus se distingue d'abord en offrant des fondations pieuses des comtes de Castille l'évocation la plus complète. Ferrand Gonzalez, Garsias Fernandez, Sanche Garsias : ce sont là les trois gouvernants les plus anciens dont des chartes de fondations monastiques aient été conservées. Quant au contenu de ces évocations, il est particulièrement riche: fondateur, saint patron, localisation, dotation, mention sépulcrale, en une occasion déclaration du statut d' "infantat "21. C'est que dans l'oeuvre du Tolédan, fondations et inhumations participent pleinement, et jusque dans l'organisation formelle profonde du propos, d'une représentation de la genèse d'un territoire castillan, d'un espace doté

\footnotetext{
21 "Monasterium Sancti Petri in ripa Aslantiae fluminis aedificavit [Ferdinandus Gundisalvi], et multis possessionibus illud dotavit. Morte propia defunctus, in eodem monasterio est sepultus ", De rebus, p. 99a ; " [Garsias Ferdinandi] construxit monasterium in honorem Sancti Cosmae et Damiani iuxta ripam Dorii fluminis Aslantiae, in villa quae Cavae rubeae nuncupatur, et amplissimis possessionibus dilatavit, et loca quae dedit, statuit Infantaticum appellari eo intuitu, quod si aliqua de genere suo non posset, aut nollet mariti comparis solatia adipisci, de bonis monasterii provideretur eidem largiter et decenter, demptis dumtaxat necessariis clericorum qui inibi Deo et Sancti Martyribus deservirent", ibid., p. 99a. La fondation du monastère d'Oña est, historiquement, moins précise. En revanche, elle inspire une légende de grande conséquence pour ce qui nous occupe et que j'étudierai plus loin. Je reproduis, dès cette note, l'entier du récit : " [Sancii comitis] mater optans commercium cuiusdam Principis Sarraceni, proposuit filium interficere, ut sic cum munitionibus et oppidis optatis nuptiis potiretur. Cumque quodam sero letali poculo virus mortiferum miscuissat, filius revelatione pedissequae hoc praesensit, et matri, ut prius biberet, supplicavit. Quod ipsa renuens, demum coacta, quod male miscuerat, degustavit, et parricida mater hausit, et meruit mortem in poculo quod paravit. Et tandem Comes Sancius, contriti cordis poenitentia stimulatus, construxit monasterium nobile quod Oniam nominavit, eo quod matrem viventem Mioniam more Hispanico appellabat. Demum post multas victorias strenue consummatas felicem spiritum suo retituit creatori, et in monasterio Oniensi quod ipse construxerat, est sepultus ", ibid., p. 99b-100a.
} 
d'une identité historico-politique dont Rodrigue, dans le champ de l'historiographie royale, est l'inventeur ${ }^{22}$.

Le libre V de l'Historia de rebus Hispaniae s'ouvre sur l'élection des juges de Castille $^{23}$. Modo Toletano, cette légende, alors vieille seulement d'un demi-siècle et dont le Liber regum puis le Chronicon mundi avaient offert les deux premières versions, au demeurant fort divergentes, rapporte comment, sous le règne de Fruela II (894-895 selon Rodrigue, en fait 924-925), les Castillans, en butte aux attaques des ennemis et à l'injustice des rois de León, qui étaient alors leurs rois, se dotèrent d'une instance judiciaire autonome en élisant deux juges, Nuño Rasura et Laïn Calvo, dont le premier fut à l'origine du lignage des comtes puis des rois de Castille. De l'intention des électeurs, le De rebus déclare : “iudices statuerunt ut dissenssiones patriae et querelantium causae eorum iudicio sopirentur "24. De Nuño Rasura, 1'on saura seulement qu'il rend des “iudicia"25. L'institution des juges semble donc un aménagement juridictionnel sans conséquence capitale sur la dépendance des Castillans à l'endroit des rois de León. Il s'agit néanmoins, au regard de la justice royale léonaise, d'une aliénation tout à fait considérable et l'institution est faite pour durer: " sibi et posteris providerunt $" 26$, écrit le Tolédan.

L'événement est localisé. Les électeurs sont, non pas tout à fait les Castillans, comme je viens de l'écrire, mais l'élite de leurs ancêtres, les “ nobiles Barduliae, quae nunc Castella dicitur "27. La dissidence émane donc d'un espace, distingué et identifié par un nom ("Bardulia") et qui sera soumis désormais à une juridiction autochtone. La définition de cet espace relève d'un référent ethnique. La Bardulie est la terre des

\footnotetext{
${ }^{22}$ Bien entendu, cette "inventio" ne vaut que pour le champ de l'historiographie royale et de ses représentations. Le cartulaire castillan, qui n'a pas été suffisamment étudié dans ce sens, porte un témoignage du plus grand intérêt, quoique d'un autre type, sur la conscience historique qu'avaient les Castillans de la genèse de leur territoire. Cette étude voudrait répondre, bien modestement et sous le seul rapport d'une représentation du territoire, au voeu de Salvador de MOXÓ, qui souhaitait un " examen attentif de la chronique du Tolédan" en ce qui concerne les premiers pas de l'indépendance politique de la Castille [" Castilla ¿principado feudal ?", Revista de la Universidad de Madrid (Homenaje a Menéndez Pidal, 3), 19 (73), 1970, p. 229-257, p. 235].

${ }^{23}$ Sur cette légende, voir mon livre mentionné en note 1 et, pour une étude plus fouillée de la version imaginée par Rodrigue de Tolède, les pages 251 à 316.

${ }^{24}$ De rebus, p. $97 \mathrm{~b}$.

${ }^{25}$ Ibid., p. 98a : "vix esset cui eius iudicia displicerent".

${ }^{26}$ Ibid.

${ }^{27}$ Ibid., p. 97a.
} 
Bardules $^{28}$, au même titre que les Asturies sont le territoire dominé par les Asturs. De Garsias Fernandez, arrière-petit-fils de Nuño Rasura, Rodrigue dira encore "bella plurima contra Reges Asturiarum [...] gloriose peregit "29. Il y avait pourtant belle lurette que les rois contre lesquels s'affirmait alors la souveraineté castillane s'intitulaient « rois de León »-définition spatiale, territoriale-et la Légende des juges indiquait bien que c'était à León que les Castillans essuyaient les vexations des rois et des magnats : " eis euntibus ad iudicium, a Regibus et magnatibus Legione iniuriose fiebant $»^{30}$. La documentation atteste pourtant l'emploi du nom "Castella" -terre, région des châteaux- dès l'an $800^{31}$ et, pour le même épisode, la principale source de Rodrigue, le Chronicon mundi, mettait face à face les "nobiles de Castella" et le "regnum legionense" 32 . Fidèle à 1'Historia silensis ${ }^{33}(c$. 1150) et, en-deçà, à Sampire (fin $\mathrm{X}^{\mathrm{e}}$ siècle) et à la Chronique d'Alphonse III (fin $\mathrm{IX}^{\mathrm{e}}$-début $\mathrm{X}^{\mathrm{e}}$ siècle), Luc utilisait pour la dernière fois le mot "Bardulia", cinq règnes avant celui de Fruela II, aux premiers jours du règne de Ramire Ier (a. 842) : “ in Barduliam, quae nunc Castella dicitur, perrexerat ad accipiendam uxorem "34. Rodrigue lui-même, rapportant, à la fin du quatrième livre, la capture à Téjar puis l'exécution de certains comtes castillans par Ordoño II -cet événement est présenté comme une des causes de l'élection des juges de Castille-, écrivait, suivant pour une fois scrupuleusement sa source léonaise : “ comites qui Castellam regere videbantur " ${ }^{\prime 35}$. Pourtant, le récit légendaire prolonge l'existence de la Bardulie jusqu'à l'institution des juges Nuño Rasura et Laïn Calvo, et lorsque le Tolédan justifie le geste des électeurs par le harcèlement belliqueux de leurs frontières,

${ }^{28}$ Claudio SÁNCHEZ-ALBORNOZ, "Divisiones tribales y administrativas del solar del reino de Asturias en la época romana", Boletín de la Academia de la Historia, 95, 1929, p. 351 sq. ainsi que "El nombre de Castilla ", in : Estudios dedicados a don Ramón Menéndez Pidal, 7 t. en 8 vol., Madrid : CSIC, 1950-1962, p. 629-641.

${ }^{29}$ De rebus, p. 99a.

${ }^{30} \mathrm{Ibid}$., p. $97 \mathrm{~b}$.

${ }^{31}$ Sa fréquence d'emploi est grande dès les premières décennies du IXe siècle (cf. SÁNCHEZALBORNOZ, "El nombre de Castilla ", cité en note 28).

${ }^{32}$ Chronicon, p. 82 et 83.

${ }^{33}$ Francisco SANTOS COCO, Historia silense, Madrid : JAEIC, 1921, p. 28 : “ Ceterum Ranimirus adulta iam etate, cum Bardulies, que nunc Castella vocatur, ad accipiendam uxorem accederet...". Chronique d'Alphonse III, in: Yves BONNAZ, éd., Chroniques asturiennes (fin IXe siècle), Paris: Éditions du CNRS,1987, p. 53: "in Barduliensem prouinciam fuerat aduectus ad accipiendam uxorem"; on trouve dans la liste des contrées peuplées sous Alphonse Ier "Bardulies quae nunc appellatur (/uocitatur) Castella", ibid., p. 46. Les textes les plus anciens écrivent le nom au pluriel, semblablement à " Asturies". On trouve également "Bardulias" dans les Annales compostellani : "Era DCCCXXX. Venit Abutaman in Alabam mense tertio, qui \& occisus fuit Era DCCCXLIIII. in Pisuerga, quando venit in Bardulias", FLÓREZ, 23, p. 319.

${ }^{34}$ Chronicon, p. 76.

${ }^{35}$ De rebus, p. 96b. La source est le Chronicon mundi (Chronicon, p. 82). 
c'est encore en termes ethniques qu'il délimite celles-ci : “ videntes etiam quod termini gentis suae ex omnibus partibus arctabantur... "36

Rodrigue substitue ainsi à la conception territoriale de la domination exercée par la royauté léonaise sur la Castille, qui était celle de ses contemporain -un comté ou un ensemble de comtés inclus dans un royaume- une vision plus ancienne, ressortissant à la nuit des premières ethnies hispaniques: l'hégémonie des Asturs sur les Bardules. L'inclusion sur laquelle reposait la conception dominante du rapport politique de la Castille avec le royaume de León est ainsi écartée au profit d'une dissociation d'espaces rattachés à des ethnies distinctes. Et le territoire castillan se trouve désormais doté d'un enracinement ethnique originaire de même rang que les Asturs pour le León, ou, pour la Navarre, les Vascons ${ }^{37}$. Pourvue de ce soubassement ethnique dissocié et d'une juridiction autochtone, la Castille peut naître. Du reste, son nom apparaît dès la première décision de Nuño Rasura : "Nunius... fere ab omnibus Castellae militibus domicellos filios petiit nutriendos "38. La juridiction fait donc le territoire et la Castille, dans un premier temps, est le lieu de vie d'un peuple qui a accédé à une forme d'autonomie juridictionnelle, la conséquence d'un acte par lequel une "gens" se dote d'une institution dont les compétences sont certes seulement judiciaires mais qui n'en est pas moins déjà de nature politique dans la mesure ou elle déplace la juridiction des rois "des Asturies".

De l'espace castillan, pour l'instant, nous ne saurons pas davantage. Nous n'en apprendrons pas beaucoup plus dans les lignes consacrées au fils de Nuño Rasura, Gonzague Nuñez, dont il est dit toutefois: "multa enim strenue contra Arabes

\footnotetext{
${ }^{36}$ De rebus, p. $97 \mathrm{~b}$.

${ }^{37}$ Selon Rodrigue, les premiers combats de la "reconquête" sont menés sous le commandement d'un Goth, ancêtre fondateur de la dynastie royale " néo-wisigothique". Mais le gros des hommes que conduit Pélage est constitué d'Asturs. C'est seulement après la défaite de Munuza, seigneur musulman de Gijón, que les Goths d'Al-Andalus, encouragés par ce revers de l'occupant, rejoignent en nombre les rangs des troupes astures : "Et dum tot dispendiis Hispaniam dissecarent, Deus omnipotens in ira sua misericordiae non oblitus, Pelagium quasi scintillam modicam in suo conspectu voluit conservare. Hic Pelagius (ut est dictum) fugiens a facie Vitizae qui eum voluerat excaecare, licet Spatharius eius fuisset [...] Asturiis se donavit, ut saltem in Asturiarum angustiis posset christiani nominis aliquam scintillam conservare. [...] Inveniensque plurimos qui metu ad edicta Arabum properebant, spiritu fortitudinis et spe in Domino confortavit, quia etsi propter peccata flagellat filios pestilentes, in fine tamen non obliviscetur misereri. Illi autem sacra monita attendentes, excusso metu facti magnanimi et fideles, ad montem magnum qui Auseva dicitur, conscenderunt, et per omnes Astures sacra monita persuadens, quasi de gravi somno pusillanimes excitavit, et de omnibus partibus Asturiarum ad eum tamquam ad Dei nuncium concurrentes, in tanta desolatione ipsum in Principem elegerunt. [...] Munuza qui civitati Gegioni maritimae et Asturiis praesidebat, fugae periculo se commisit : sed ab Asturibus in vico qui Olalies dicitur, fuit captus et interfectus. [...] Gothi autem quibus fugae facultas afuerat, audientes quod a Gothis manus Domini non discesserat, clanculo se furantes, ad Pelagium Principem advenerunt, et zelantes legem, et Machabaeorum iustitiam aemulantes, pro fide commori elegerunt" (De rebus, IV, 1, p. $74-75$ et IV, 4 , p. 78b).

${ }^{38}$ Ibid., p. 98b.
} 
peragendo, fines patriae ampliavit "39 . C'est que les fonctions exclusivement judiciaires du premier juge ont été, dans le cas de son successeur, augmentées par la concession du “principatum militiae "40. L'expansion est ainsi liée à l'instauration d'un commandement militaire unique en Castille, mais elle accompagne aussi l'attribution à un même homme et pour l'ensemble du territoire du pouvoir judiciaire et du pouvoir militaire dont la réunion fondait habituellement le pouvoir politique. Genèse du territorial et genèse du politique sont donc, dans l'opinion de Rodrigue, strictement concomitantes.

C'est pourquoi, sans doute, il faut attendre l'érection en comte du fils de Gonzague Nuñez, Ferrand Gonzalez, par l'ensemble des Castillans -“tam a magnatibus et militibus quam ab universis populis castellanis in comitem crearetur"- et la soumission de tous à son pouvoir - "et omnes se suae subiicerent ditioni" ${ }^{41}$-, c'est-à-dire l'attribution d'un titre et la reconnaissance d'une seigneurie, pour que l'espace castillan devienne plus visible. Ce progrès, du reste, est salué par le premier emploi du mot "dominium" pour qualifier le pouvoir comtal tandis qu'une métaphore sibylline pourrait indiquer que les Castillans se considèrent désormais libérés de la tutelle politique des rois " des Asturies" :

" [Ferdinandus Gundisalvi] factus comes, totam Castellam sic pacifico dominio confovebat, ut omnes Deo gratias agerent, qui per talem comitem a populo suo relevaverat sarcinam servitutis $", 42$.

«A populo suo »: émergence capitale du «populus » castillan et savante culture politique de Rodrigue Jimenez de Rada ${ }^{43}$. Alors que l'existence de la Bardulie tenait tout entière à l'implantation géographique des Bardules, la «gens » induisant le territoire, c'est, à l'inverse, l'achèvement politique du territoire castillan, couronné par son accession à l'indépendance, qui sont fondateurs d'un "populus ». Aussitôt apparaissent des noms de villes ou de places fortes conquises par les " chrétiens" sur les

\footnotetext{
${ }^{39}$ Ibid., p. 98b.

40 " [...] patre suo mortuo, patri fuit favore omnium substitutus, et etiam principatum militiae [...] addiderunt", ibid., p. 98 b.

${ }^{41}$ Ibid., p. 98b.

${ }^{42}$ Ibid., p. 98b. Le propos de Rodrigue n'est pas sans analogie avec ces mots précoces de la Chronica najerensis : "[Ferdinandus Gonzaluez] qui castellanos de sub iugo Legionensis dominationis dicitur extrasisse" (UBIETO, p. 90).

${ }^{43}$ On se souvient du De republica de Cicéron $(1,25,39)$ : « Est igitur, inquit Africanus, res publica, res populi : populus autem non omnis hominum coetus quoquo modo congregatus, sed coetus multitudinis iuris consensu et utilitatis communione sociatus ».
} 
"Arabes" -Osma et, dominant le Duero, San Esteban: "Hic contra Arabes plurima bella gessit, [Oxomam] et Sanctum Stephanum et alia plurima loca christianae restituit ditioni " 44 - tandis qu' une frontière fluviale se dessine à l'ouest entre la "Castille " et les "Asturies":

"Ex quo iste suscepit suae patriae comitatum, cessaverunt reges Asturiarum insolescere in Castellam, et a flumine Pisorica nihil amplius vindicarunt ${ }^{\$ 4}$.

C'est ce processus de construction politique et de délimitation territoriale que semble couronner la première fondation pieuse, celle de Saint-Pierre d'Arlanza, puis la mort et l'inhumation du comte dans le monastère :

"Monasterium Sancti Petri in ripa Aslantiae fluminis aedificavit, et multis possessionibus illud dotavit. Morte propria defunctus, in eodem monasterio est sepultus $" 46$.

Ainsi, le territoire castillan se précise au fur et à mesure qu'y éclôt un pouvoir autochtone qui, naissant du "judicatus " 47 , se voit bientôt complété par le " principatum militiae" et s'épanouit enfin dans le "dominium" d'un «comes» souverain. La géographie politique de ce territoire consiste en des places fortes ou des villes avancées, un fleuve frontière, un lieu de prière et de mémoire. La genèse du territoire est donc constamment rapportée aux éléments d'une représentation complexe du social: la construction du pouvoir, l'expansion militaire, le peuplement, l'affirmation religieuse et l'aspiration spirituelle, la mémorisation du passé. Dans l'érection en comte de Ferrand Gonzalez s'esquisse même une structure de la société laïque (" magnates", " milites", "populi"), tandis que la maturité politique de la Castille et son accession à l'indépendance se soldent par l'avènement d'un «populus ». Deux grandes oppositions catégoriques dominent toutefois la délimitation de l'espace territorial : une division religieuse entre chrétiens et "Arabes", une division politique entre la Castille et "les Asturies". La fondation monastique et l'inhumation comtale me paraissent dépendre de

\footnotetext{
${ }^{44}$ De rebus, p. $98 \mathrm{~b}-99$ a.

${ }^{45}$ Ibid., p. 98b-99a. Erreur de copie dans l'édition Lorenzana (“ quoniam" pour "Oxomam ”). Voir Rodrigo JIMÉNEZ DE RADA, Historia de los hechos de España, (Juan FERNÁNDEZ VALVERDE, éd.), Madrid : Alianza Editorial, 1989, p. 193. Le processus est plus rapide dans le Chronicon mundi où, dès la magistrature de Nuño Rasura, Luc de Túy déclare: "Tunc enim angustatum est regnum Legionense \& in praedicto flumine metam fecit" (Chronicon, p. 83).

${ }^{46}$ De rebus, p. 99a.
} 
ces deux grandes catégories, le religieux et le politique -comme du reste de deux grands ordres, le spirituel et l'historique-, de la perception du réel.

Le gouvernement de Garsias Fernandez reconduit, pour l'essentiel, le système que l'on voit se mettre en place sous celui de son père. Une ligne fortifiée marque maintenant la limite méridionale constituée par le Duero -" castra plurima in ripa Dorii munifice reparavit..."- tandis que la frontière occidentale de la juridiction castillane, soldant de nombreux combats frontaliers avec les rois " des Asturies" -" bella plurima contra Reges Asturiarum conantes Castellae finibus praeeminere gloriose peregit "48 - et préparant l'incorporation de toute la Terre de Campos ${ }^{49}$, $s^{\prime}$ avance désormais jusqu'au Carrión : " [...] et usque ad fluvium Carrionem iurisdictionis suae terminos dilatavit " Ici encore, la fondation et la dotation d'un monastère, celui de Saint-Côme et SaintDamien de Covarrubias, situé, comme celui de Saint-Pierre, sur l'Arlanza, parachèvent la consolidation et l'expansion du territoire. Cet établissement, toutefois, n'accueillera pas, comme les deux autres, la dépouille de son fondateur, dont Rodrigue tient peut-être des Annales compostellani qu'il fut enterré à Saint-Pierre de Cardeña ${ }^{51}$. Comme par compensation, l'auteur évoque une mission nouvelle du monastère. L' « infantaticum » apparaît, destiné à accueillir la part féminine du lignage comtal vouée, volontairement ou involontairement, au célibat ${ }^{52}$. Rodrigue n'en dit pas davantage sur la fonction et le sens de l'infantazgo -sur, éventuellement, la "part divine" du pouvoir comtal que pourrait représenter, selon Patrick Henriet ${ }^{53}$, cette composante féminine Deo vota du lignage. Si Rodrigue a disposé de la charte de fondation du monastère de Covarrubias, il s'en écarte ici. La charte, en effet, se borne à déclarer la donation d'Urraque, fille du comte et de la comtesse, à Jésus-Christ et à ses saints et la donation à Urraque, sous forme d'une seigneurie assez indépendante, du monastère et de très nombreuses

\footnotetext{
${ }^{47}$ J'emprunte le terme à Luc de Túy qui, parlant de Nuño Rasura, déclare: "Sapienter se gessit Nunnus Rasoira in iudicatu suo" (Chronicon, p. 82).

${ }^{48}$ De rebus, p. 99a.

${ }^{49}$ La possession des contrées situées entre les rivières Cea et Pisuerga est longtemps un sujet de conflit entre le León et la Castille. Il trouve de multiples expressions dans l'historiographie [cf. G. MARTIN, Les Juges de Castille..., p. 217-218 et Histoires de l'Espagne médiévale. Historiographie, geste, romancero, Paris : Klincksieck, 1997, p. 57-68 et notamment p. 61].

${ }^{50}$ De rebus, p. 99a.

51 "Obiit autem morte propria, et in monasterio Santi Petri de Cardonia tumulatur ", ibid., p. 99a.

${ }^{52} \mathrm{Cf}$. texte cité en note 21 .

${ }^{53}$ Patrick HENRIET, "Deo votas. L'infantado et la fonction des infantes dans la Castille et le León des Xe-XIIe siècles ", Cultures et civilisations médiévales, 23, 2000 [Au cloître et dans le monde. Femmes, hommes et sociétés (IXe-XVe siècles). Mélanges en l'honneur de Paulette L'Hermite-Leclercq, (Patrick HENRIET et Anne-Marie LEGRAS, éd.)], p. 189-203.
} 
possessions $^{54}$. Certes, notre historien interprète la fondation de Covarrubias avec le savoir de celui qui a vu se pérenniser l'infantazgo. Dans la formation du territoire castillan, il rend néanmoins l'association de l'univers religieux et de l'univers politique plus que jamais efficace puisque, à défaut d'être la sépulture et la mémoire de son fondateur, ce monastère se voit destiné à renfermer indéfiniment une part vivante et, comme l'a montré Patrick Henriet et le savait Rodrigue, fort agissante de la parenté des comtes et, au-delà, des rois de Castille.

Sanche Garsias n'est pas le dernier représentant mâle de la lignée comtale. Cependant, comme son fils et jeune successeur Garsias Sanchez meurt sans postérité et que le comté castillan passe alors sous l'influence du roi de Navarre Sanche III le Grand, époux de la soeur de Garsias, l'infante Elvire, son gouvernement donne lieu à un chapitre réservé et marque le couronnement et la clôture de l'évocation chaînée des premiers comtes de Castille ${ }^{55}$. À sa suite, le propos de Rodrigue reprendra le cours, interrompu en amont, des règnes léonais. Au titre de la représentation du territoire castillan, ce troisième chapitre du livre V du De rebus offre un relief particulier. Outre une très longue liste de villes ou de places fortes reprises par Sanche aux Sarrasins Peñafiel, Sepúlveda, Montejo, Gormaz, Osma et San Esteban : en gros, toute la ligne du Duero perdue sous Garsias Fernandez ${ }^{56}$-, outre la fondation du monastère d'Oña, dont il est dit que le comte y fut ensuite enseveli ${ }^{57}$, nous trouvons ici la première évocation d'une activité proprement législative avec la concession d'une charte municipale et, plus important encore pour le thème qui nous concerne, d'une mesure affectant l'ordre politique et social de l'ensemble du comté. Le comte Sanche -dont la Chronica

\footnotetext{
54 “ Decrebimus munus offere Domino Ihesu Christo et Sanctis eius, id est prolem filiamque nostram Urraca et elegimus ipsius loci que Couerubensis situm, qui extat in ripa fluminis Asilancas, reliquie namque residentis loci illius sanctorum Cosme et Damiani et apostoli et sanctorum Iusti et Pastoris, testium Christi. Qua quidem, ego Garsea Fredinandez comite et Aua comitissa donamus tibi, filia nostra Urraca, in Couarubias cum suis terminis, uidelicet, de semitario antiquo de Mambulas usque in ualle de Sancto Petro, qui descendet de serra sicut aqua discurret usque in Aslanca... ", ZABALZA DUQUE, op. cit., p. 398.

${ }^{55}$ Ces mouvements dynastiques structurent très consciemment le propos de Rodrigue de Tolède, $c f$. chapitre 21 du livre V (De rebus, p. 112a).

${ }^{56}$ La liste de l'édition Lorenzana est un peu plus longue et parfois obscure: “ Hic obtinuit Pennam fidelem, et Septempublicam, et Madolium [/Malvolium], et Montelionem, et Varinatium, Oaromam [/Oxomam], et Sanctum Stephanum, quae in captionem patris perdiderant Christiani... ", ibid., p. 99b. Les annales castillanes et de la Rioja citaient Gormaz, Osma, San Esteban "et alias casas in Estremadura" (Annales castellani II, GÓMEZ-MORENO, p. 26 ; Annales compostellani, FLÓREZ, 23, p. 320 ; Annales tolédanes I, ibid., p. 385. Même chose pour Luc de Túy (" Hic accepit a Sarracenis Gormaz, Osmam \& Sanctum Stephanum, \& alia multa in Extremadura ", Chronicon, p. 90). Tous les Montejo que je connaisse se trouvent en effet au sud du Duero.

${ }^{57} \mathrm{Cf}$. note 21.
} 
najerensis, les Chroniques navarraises brèves, le Liber regum, les premières Annales tolédanes et même Luc de Túy ${ }^{58}$ disaient certes qu'il avait “donné les bons fors"- est ici réputé avoir concédé les "antiquos foros Septempublicae" (les fors anciens de Sepúlveda $)^{59}$ et avoir renforcé les " libertés" de la chevalerie castillane : "Castellanis militibus qui et tributa solvere et militare cum principe tenebantur, contulit libertates, videlicet ut nec ad tributum aliquod teneantur nec sine stipendiis militare cogantur $" 60$.

L'activité législative du comte Sanche, dont la précision contraste avec l'image systématique mais floue que Rodrigue donnait jusqu'ici des progrès institutionnels de la Castille, est comme l'aboutissement et la concrétisation ponctuelle d'un ordre dont les caractéristiques apparaissent par petites touches depuis la fondation de la magistrature originaire. Cet ordre, dont le De rebus manifeste par d'autres nombreux traits, qu'il est cher à Rodrigue Jimenez de Rada, tient pour l'essentiel en l'idée selon laquelle le salut du royaume -comme celui d'un comté qui le préfigure- repose sur l'entente harmonieuse du prince avec le groupe aristocratique. Outre un ensemble de valeurs politiques consensuelles dont Rodrigue pare le bon gouvernant ("prudentia", industria", “ circonspectio", “curialitas", “affabilitas"), un grand schéma de réciprocité entre la "largitas" du prince et la "fidelitas" des chevaliers, donnant lieu à un affect nommé " amor", "caritas" ou “ dilectio" régit la vision politique du De rebus, empruntant quelquefois sa terminologie, sans toutefois prendre celle-ci véritablement pour modèle, à la féodalité française ${ }^{61}$.

L'accord entre la noblesse et la magistrature suprême est intrinsèque à l'institution des juges, dont l'élection est décidée et réalisée par les “ nobiles Barduliae ". Il marque aussi le portrait et le gouvernement du bien-aimé Nuño Rasura (" ab omnibus amabatur"), dont les vertus ("patiens et modestus "62), la pratique judiciaire, où la composition amiable prévaut sur la sentence -“ vix esset cui eius iudicia displicerent, aut

\footnotetext{
${ }^{58}$ Chronica najerensis : UBIETO, p. 91 ("Comes Santius cognomento bonus, pro eo quod bona fora dedit ") ; Chroniques navarraises : UBIETO, p. 39 (" el conde de [sic] don Sancho, qui los bonos fueros dio ") ; Liber regum: COOPER, p. 33 (" [e]l comte don Sancho, el qui dio los buenos fueros"); Annales tolédanes I: FLÓREZ, 23, p. 385 (" el Conde D. Sancho, el que dio los buenos fueros"); Chronicon, p. 90 ("Dedit namque bonos foros \& mores in tota Castella").

${ }^{59}$ De rebus, p. 99b. Fuero de Sepúlveda in : Tomás MUÑOZ y ROMERO, Colección de fueros municipales y cartas pueblas, Madrid : Imprenta de don José María Alonso, 1847 (fac-similé, Madrid : Atlas, 1978), p. 281-286.

${ }^{60}$ De rebus, p. $99 \mathrm{~b}$.

${ }^{61}$ Là-dessus, MARTIN, Les Juges de Castille..., p. 260-270 et bientôt «Noblesse et royauté dans le De rebus Hispaniae (livres 4 à 9) " à paraître dans les Cahiers de linguistique et de civilisation hispaniques médiévales, 26, 2003. Sur la "curialitas", Adeline RUCQUOI, "La royauté sous Alphonse VIII de Castille ", Cahiers de linguistique hispanique médiévale, 23, 2000, p. 215-241.
} 
eius sententias causaretur, quas tamen rarissime proferebat, quia in compositione amicabili fere omnia terminabat ${ }^{" 63}$-, et, plus largement, les initiatives politiques, comme celle de former les enfants de la " chevalerie" castillane à la courtoisie et aux bonnes moeurs de telle manière que les " patres adolescentium de profectus filliorum profiterentur se tali nutricio obligatos ${ }^{~} 64$, répondent aussi bien aux attentes de la noblesse de Castille qu'aux intérêts du magistrat. La pérennité dynastique du pouvoir dans la lignée du premier juge est elle-même la conséquence d'une histoire d'amour entre son fils Gonzague Nuñez et les nobles adolescents dont son père l'a entouré :

\footnotetext{
"ipsi adolescentes sic erant Gundisalvo Nunii dilectione coniuncti, ut eum quasi dominum sociarent, nec possent ab eius consortio vel ad modicum separari ${ }^{~} 65$.
}

Quant à Ferrand Gonzalez, nous l'avons vu érigé en comte par l'élan commun des Castillans à la tête desquels, néanmoins, s'étaient portés magnats et chevaliers. De tout ceci, la représentation n'est certes pas absente d'une entente idyllique autant qu'abstraite entre gouvernant et gouvernés, entre le seigneur des Castillans et l'ensemble de ses sujets, représentation dont, au demeurant, le comte Sanche " in subditos totus pius "66 est le meilleur exemple, dont Rodrigue écrit d'une part, pensant peut-être aux franchises concédées à la chevalerie, que "nobiles nobilitate potiore donavit" et d'autre part, pensant peut-être aux fors municipaux de Sepúlveda, que "in minores servitutis duritiam temperavit" ${ }^{67}$. Les lois édictées par Sanche en faveur de la noblesse n'en demeurent pas moins celles dont le De rebus manifeste avec le plus de précision le contenu.

La genèse du territoire castillan associe donc ici trois processus : un processus de dissociation et de conformation politique, un processus de consolidation et d'expansion militaire, un processus d'investissement spirituel et monumental. Il est bien intéressant, pour la connaissance de Rodrigue de même sans doute que pour celle des mentalités castillanes de la première moitié du XIIIe siècle ${ }^{68}$, que, dans la genèse du territoire et de

\footnotetext{
${ }^{62}$ De rebus, p. 98 a.

${ }^{63}$ Ibid., p. 98a.

${ }^{64}$ Ibid., p. 98 b.

${ }^{65}$ Ibid., p. $98 \mathrm{~b}$.

${ }^{66}$ Ibid., p. 99b.

${ }^{67}$ Ibid., p. 111a.

${ }^{68}$ Dans le Chronicon mundi les fonctions de Nuño Rasura semblent, de même, exclusivement judiciaires (" Sapienter se gessit Nunnus Rasoira in iudicatu suo, \& totam Castellam usque flumen de Pisorga iudicavit dum vixit ") tandis que son fils Gonzague Nuñez exerce conjointement des fonctions
} 
son autorité politique, la fondation d'une instance judiciaire précède naturellement celle d'un commandement militaire et que, dans la lignée de Nuño Rasura, la détention du “judicatus" soit antérieure d'une génération à celle du "principatum militiae". La genèse d'un pouvoir politique castillan est, quoi qu'il en soit, le lieu par excellence de l'endoctrinement du récit : Rodrigue Jimenez de Rada y manifeste ses convictions, qui sont peut-être celles d'une partie de l'entourage de Ferdinand III ou de la reine mère Bérengère. Le second processus, de nature militaire, est celui dont l'incidence est la plus directe sur la représentation d'un espace. Il donne lieu à l'indication de peuplements souvent fortifiés, et surtout de frontières dont le choix est néanmoins dicté par les deux autres processus puisqu'elles relèvent, à l'ouest, d'une séparation politique d'avec le León et, au sud, d'un antagonisme religieux avec les Arabes. Le troisième processus, que traduisent les fondations monastiques, est celui dont la mise en œuvre est la plus tardive. Sa manifestation coïncide avec la pleine maturité d'un pouvoir politique castillan, le " judicatus", bientôt associé au " principatum militiae ", ayant donné le jour au "dominium" comtal et une juridiction autochtone s'étant transformée en comté indépendant. Le début des fondations pieuses marque donc le terme de cette évolution. Ce processus collabore néanmoins, au même titre que les autres, à la différenciation et à l'identification du territoire. Face aux "Arabes", dont le De rebus montre régulièrement, à partir de Gonzague Nuñez, les combats que leur livrent les juges puis les comtes de Castille, il pose d'abord l'existence d'un territoire chrétien proprement castillan. Ensuite et surtout, le chapelet des fondations monastiques des comtes de Castille, qui sont presque autant de sépultures, fait pendant et fait front aux fondations et aux ensevelissements des rois asturs-léonais, les seuls que l'historiographie léonaise se complaisait à rappeler et que Rodrigue, au demeurant, reprend sans exception dans ce même cinquième livre: Saint-Julien, Saint-Sauveur de León, Saint-André et SaintChristophe, Sainte-Marie, Saint-Michel-Archange.

Un mot assemble dans le De rebus ces divers aspects d'une genèse et d'une identification territoriales de la Castille en même temps qu'il les interprète dans la représentation affective d'une appartenance. Ce mot, dont on aura noté l'emploi au fil de mes citations, est celui de "patria ". Au sens de "pays d'origine" d'un homme, de “pays des ancêtres", le mot n'est pas inconnu de l'historiographie espagnole antérieure 
à Rodrigue ${ }^{69}$, bien qu'il y soit rare, et Luc de Túy, à l'occasion, l'utilise savamment plutôt, du reste, en référence à l' "Hispania "70 " Patria " distingue toutefois ce segment historique du De rebus par sa fréquence, son application et son sens. Alors que dans le livre IV, pourtant consacré aux premiers pas d'une royauté chrétienne post-wisigothique en Asturies et en León, moment-clé, s'il en est, de la tradition historiographique espagnole médiévale, il est employé quatre fois, pour dénoter d'abord l'ancienne Espagne wisigothique puis le royaume astur-léonais, on compte dans le cinquième livre neuf occurrences de "patria" toutes réservées à la Castille ${ }^{71}$. La “patria” est un espace de justice et de paix :

"duos milites [...] elegerunt quos et iudices statuerunt ut dissensiones patriae $[\ldots]$ eorum iudicio sopirentur ",

"Cumque [Gundisalvus Nunii] crevisset factus miles militiam strenuus exercebat et pacis dulcedinem in patria retinebat $"$ "72.

La “ patria " est un espace gagné, défendu ou agrandi par l'action militaire :

"[Gundisalvus Nunii] fines patriae ampliavit",

"vir strenuus Fernandus Gundisalvi comes Castellae moritur, qui in acquisitione et tuitione et dilatatione patriae utiliter, strenueque, et fideliter laborarat $"$ "73.

La " patria" est enfin, l'espace politique et religieux castillan défini par opposition aux Asturies et aux Arabes :

"Ex quo iste suscepit suae patriae comitatum, cessaverunt reges Asturiarum insolescere in Castellam et a flumine Pisorica nihil amplius vindicarunt:

\footnotetext{
${ }^{69} \mathrm{Il}$ est employé dans ce sens, à quelques rares reprises, dans l'Historia silensis (SANTOS COCO, p. 10 et 26, par exemple).

${ }^{70}$ Dans le Chronicon aussi l'emploi est rare, mais il arrive que le sens comporte une nuance plus affective et communautaire : " pro patria \& fide Catholica fortiter dimicarunt " écrit par exemple Luc des chrétiens qui résistent à l'assaut d'Abdamelech (Chronicon, p. 88). Les armées chrétiennes rassemblent toutefois Léonais, Navarrais et Castillans. Luc, il est vrai, est un grand nostalgique de $l^{\prime}$ Espagne gothique (et isidorienne...). Sur l'usage et la signification le plus souvent " hispanique " du mot dans l'oeuvre complète de Luc, voir Patrick HENRIET, "Sanctissima patria. Points et thèmes communs aux trois oeuvres de Lucas de Túy", Cahiers de linguistique et de civilisation hispaniques médiévales, 24, 2001, p. 249-278.

${ }^{71}$ Salvador de MOXÓ avait déjà noté ce fait, op. cit., p. 236.

${ }^{72}$ De rebus, respectivement $\mathrm{p} .97 \mathrm{~b}$ et $98 \mathrm{~b}$.

${ }^{73} \mathrm{Ibid}$., respectivement p. 98b et 105.
} 
strenuitate enim sua eorum insultibus resistebat, nec propter eos a bellis Arabum desistebat" ${ }^{\text {"74 }}$.

Les occurrences et les significations du mot " patria " suivent donc bien et soulignent les arêtes de la construction d'un territoire telles que nous les avons précédemment discernées. Mais " patria" renvoie en outre et en toute première instance à un affect et aux comportements qu'il inspire. Garsias Fernandez, confronté à la révolte de son fils mais apprenant en même temps que les Sarrasins dévastent les confins du comté, préfère, " eligens mori pro patria "75, affronter les envahisseurs innombrables plutôt que de s'employer à restaurer son autorité. Quant à son fils, le comte Sanche Garsias, dont l'évocation éthico-politique ouvre le chapitre qui lui est consacré, il se trouve qualifié, non seulement de "vir virtutum" et " in subditos totus pius" mais encore d" "amator patriae $" 76$.

Or, voici que la fondation monastique attribuée à Sanche Garsias se distingue de celles prêtées à Ferrand Gonzalez et à Garsias Fernandez par un récit complexe et inconnu de l'historiographie antérieure ${ }^{77}$. Certains y ont vu la trace d'une Chanson de la comtesse félonne ${ }^{78}$, mais la tradition historiographique n'appuie pas leur thèse. Il pourrait aussi bien s'agir d'une légende locale, voire, plus plausiblement si l'on considère ses ressemblances avec le récit de la fondation de Saint-Julien par le roi Ramire II de León, “penitentia ductus " après qu'il avait fait emprisonner et énucléer son frère Alphonse ${ }^{79}$, d'une création de Rodrigue lui-même, imitant ce patron. La mère du comte Sanche, amoureuse d'un "prince sarrasin", résout d'empoisonner son fils pour donner la Castille à l'aimé. Informé de l'affaire, Sanche contraint sa mère à boire

\footnotetext{
${ }^{74}$ Ibid., p. 99a.

${ }^{75}$ Ibid., p. 111a.

${ }^{76}$ Ibid., p. 99 b.

${ }^{77}$ Ibid., p. 99b. Texte en note 21 de cet article.

${ }^{78}$ Vers 1270, l'Estoire d'Espagne accuse la comtesse Sancie, seconde femme de Garsias Fernandez, $\mathrm{d}$ 'avoir ourdi la mort de son époux, parti au combat sur un cheval qu'elle avait nourri seulement de son, puis d'avoir, comme l'écrit Rodrigue, médité par amour d'un roi maure d'empoisonner son fils Sanche. Partant de là, Menéndez Pidal, imagina l'existence d'une chanson dont la Chronica najerensis, dès 1160, se serait faite l'écho ("Relatos poéticos en las crónoicas medievales", Revista de filología española, 10 (4), 1923, p. 329-372). Menéndez Pidal allait jusqu'à estimer la longueur de l'oeuvre: “ six cents vers environ", p. 340. Or, la Najerensis, ne dit mot ni de la comtesse ni de la préparation de la mort de Garsias Fernandez ni de celle de son fils: “ [3] Comes Ferrandus Gonzaluez genuit comitem Garsiam Ferrandiz, quem rex Almazor occidit. [4] Comes Garsias Ferrandiz genuit comitem Santium, qui regem interfecit et Cordobam destruxit, et inde corpus patris sui comitis Garsia Ferrandiz transtulit Caradignam. [5] Sepultus apud Onie monasterium, quod fecerat", A. UBIETO ARTETA, p. $90-91$.

${ }^{79}$ Rodrigue consacre à cet épisode le chapitre $5 \mathrm{du}$ livre $\mathrm{V}$ du De rebus Hispaniae (De rebus, p. 100a-101a).
} 
la potion létale puis, " contriti cordis poenitentia stimulatus ", érige un monastère auquel il donne le nom par lequel il désignait sa mère "more hispanico ", c'est-à-dire en basque : Mioña (<Oña, Mère). On voit bien l'intention de l'historien-philologue. Le geste de Sanche exalte l'attachement à la "patria " par sa mise en balance, favorable à celui-ci, avec l'attachement à la mère. La fondation du monastère d'Oña, conçue comme le rachat d'un matricide "patriotique" -ou "patriatique ", pour reprendre le néologisme moins anachronique de Dominique Iogna-Prat-, consacre, par le biais d'une compensation spirituelle, la primauté de l'amour de la patrie relativement à l'attachement affectif le plus primordial.

Le livre $\mathrm{V}$ de l'Historia de rebus Hispaniae se clôt sur un troisième et dernier récit à caractère légendaire ${ }^{80}$. On en trouve les premières traces dans des oeuvres soumises à l'influence navarraise, la Chronica najerensis et le Liber regum ${ }^{81}$, où il avait pour fonction d'expliquer la naissance du royaume d'Aragon et son détachement de la Navarre. Sans doute devons-nous encore au talent de Rodrigue que ce récit justifie en outre dans le De rebus l'émergence d'une "principauté " castillane destinée à se muer très vite en royauté. Encore une fois, il met en scène une mère, une mauvaise mère quoique faussement mauvaise, cette fois. La Légende de la reine calomniée a pour protagoniste la fille du comte Sanche, "amator patriae". Selon le De rebus, Sanche avait eu un fils, Garsias, et deux filles, Elvire et Thérèse. Thérèse avait été mariée au roi Vermude III de León, Elvire, l'ânée, à Sanche III le Grand de Navarre. Après la mort du comte et à la suite de l'assassinat de l' "infant" Garsias à León, Sanche le Grand, dont la domination seigneuriale s'exerçait sur Vermude et qui se prévalait des droits que lui conférait son mariage avec Elvire, avait occupé aussitôt la Castille. Celle-ci était ainsi devenue une composante du dominium du roi de Navarre. D'Elvire, Sanche avait eu deux fils : Garsias et Ferdinand. Or, voici qu'à la suite d'une vétille toute oedipienne, Elvire ayant refusé de prêter à Garsias le cheval de son père, la reine se trouve mensongèrement accusée d'adultère par son fils. Celui-ci cherche vainement l'appui de

\footnotetext{
${ }^{80}$ De rebus, p. 116-117.

${ }^{81}$ Chronica najerensis: "Instigante namque maligni hostis uersutia, predictus Garsias non est ueritus in matrem propriam uerba proferre contumelie, et eam de adulterii iniuria diffamare. Sed Ranimirus super ipsa respondens, eam constanter et ueraciter defensauit et mendatium esse probans, de infamia et periculo liberauit. Itaque regina in tantam exarsit iram quod Garsiam maledixit, et Ranimirum intra uestes coram regali curia recipiens, et quasi parturiet illum de sub uestibus eiciens in filium adoptauit, et in regno habere fecit portionem ", UBIETO, p. 92. Liber regum : "Est rei don Sancho ouo un fillo d'otra muller, qui ouo nomne l'ifant don Remiro ; e fo muit bueno e muit esforçado, e por el saluamiento que fizo a so madrastra, la reina dona Albira,
} 
son frère cadet, Ferdinand, tandis que Ramire, fruit d'une union pré-conjugale de Sanche, se propose de défendre en combat singulier l'honneur de sa belle-mère. L'heure venue de partager le royaume, Elvire demande à son mari de léguer l'Aragon, qu'il lui avait assigné en douaire, à Ramire, ce qui provoque la naissance du royaume aragonais, mais, dans le même mouvement, elle refuse de céder à Garsias le comté dont elle avait hérité de son père et qui est transmis à Ferdinand en récompense de sa non implication dans la calomnie. Ainsi naît le "principatum Castellae" $" 82$, qui deviendra royauté lorsque, deux ans plus tard, ce même Ferdinand, à la suite de la bataille de Tamarón et de la mort de Vermude III (1037), se sera emparé de la couronne de León.

La signification de cette obtention sélective de l'héritage castillan, où le droit sert une sorte d'éthique politique -la véridicité n'est pas sans importance dans la tradition politique castillane telle que la conçoit Rodrigue, lequel, exaltant les vertus de Gonzague Nuñez, met celle d'être "in sermone verax " sur le même pied que celles d'être "in iudicio iustus" et " in militia gloriosus " ${ }^{~}{ }^{-}$, n'est pas, bien entendu, tout entière renfermée dans l'épisode de la reine calomniée. Elle ne se borne pas même à rendre compte de l'émergence d'une royauté castillane. Située en fin de chaîne, la transmission sélective de la Castille à Ferdinand par la fille du comte Sanche manifeste la continuité des valeurs en dépit de la mutation lignagère, la dynastie étant désormais navarraise. Elle se charge de tout ce qui s'est construit de sens au long d'une genèse territoriale qui a associé un espace à un ordre politique et où sont intervenues, dans un système complexe et indissociable, des représentations liées à l'ethnique, au juridique, au social et au spirituel. Dans ce système, les fondations pieuses, réceptacles des corps des premiers pères et lieu de vie des infantes sans époux, servent la culture d'une "patria" castillane en rattachant le drame des hommes à l'univers spirituel tout en inscrivant son histoire dans le sol monumental.

\footnotetext{
la muller del rei don sancho padre, diol ella sos arras, et el rei atorgo las \& ouo el reismo d'Aragon e fo rei ", COOPER, p. 37.

82 “[Rex Sancius] Fernando vero Castellae tradidit Principatum" (ibid, p. 117b).

${ }^{83}$ Ibid., p. 98b : «Hic fuit omnibus patre carior, in sermone verax, in iudicio iustus, in militia gloriosus ».
} 